\title{
Validation of names of some syntaxa of the Crimean vegetation
}

\author{
Yakiv P. Didukh (*) \& Ladislav Mucina (**, ***)
}

\begin{abstract}
Didukh, Y.P. \& Ladislav Mucina, L. Validation of the name of some syntaxa of Crimean vegetation. Lazaroa 35: 181-190 (2014).

We validate concepts and names of three alliances (Androsaco tauricae-Caricion humilis, Adonido vernalis-Stipion tirsae, Veronico multifidae-Stipion ponticae) of steppe vegetation (Festuco-Brometea) of Crimea as well as two other alliances of sub-mediterranean character (Diantho humilis-Velezion rigidae, Elytrigio nodosae-Rhuion coriariae). We also validate a number of associations, most of them to serve as the holotypes of the alliances.
\end{abstract}

Keywords: Festuco-Brometea, nomenclature of plant communities, Rhamno-Prunetea, Stipo-Trachynietea, syntaxonomy, Ukraine, yaila.

Resumen: Didukh, Y.P. \& Ladislav Mucina, L. Validación de los nombres de algunos sintaxones de la vegetación de Crimea. Lazaroa 35: 181-190 (2014).

Se validan los conceptos y nombres de tres alianzas (Androsaco tauricae-Caricion humilis, Adonido vernalis-Stipion tirsae, Veronico multifidae-Stipion ponticae) de vegetación estépica (Festuco-Brometea) de Crimea, así como dos alianzas de carácter submediterráneo (Diantho humilis-Velezion rigidae, Elytrigio nodosae-Rhuion coriariae). También se validan asociaciones, la mayoría de las cuales sirven como holotipos de dichas alianzas.

Palabras clave: Festuco-Brometea, nomenclatura sintaxonómica, Rhamno-Prunetea, Stipo-Trachynetea, Ucrania, Yaila.

\section{INTRODUCTION}

The Crimean Mountains attain the altitude of $1545 \mathrm{~m}$ asl and are stretching about $150 \mathrm{~km}$ along the coast of the Black Sea on the Crimean Peninsula. The mountain range straddles the limits of steppe and mediterranean biomes (ISACHENKO \& LaVRenKo, 1980; DidukH \& ShelyaG-SosonKo, 2003).

The steppes of the Festuco-Brometea occupy a sizable area of more than 150000 ha in Crimea. They are common on foothills of the northern macroslope of the Crimean Mts from an altitude of about $600 \mathrm{~m}$ asl to about $1500 \mathrm{~m}$ asl; the steppes are almost absent on the southern macroslope that is occupied prevalently by sub-mediterranean vegetation. Floristically these communities contain a number of Pontic and Pontic-Pannonian elements such as Agropyron cristatum subsp. pectinatum, Alyssum rostratum, Gypsophila glomerata, Iris pumila and Linum pallasianum. The majority of these species is found at lower altitudes in the Crimea. The Mediterranean and Submediterranean elements, such as Asphodeline lutea, A. taurica, Fumana procumbens, Onosma rigida and Paronychia cephalotes also play a significant role in the Crimean steppe vegetation. Nearly $10 \%$ of the species pool of the Crimean steppe is formed

\footnotetext{
* M.G. Kholodny Institute of Botany. National Academy of Science of Ukraine. Tereschenkivska 01601, Kyiv 2, Ukraine. Email: ya.diduk@gmail.com

** Iluka Chair in Vegetation Science \& Biogeography. School of Plant Biology. The University of Western Australia. 35 Stirling Highway. Crawley WA 6009, Perth, Australia. E-mail: Laco.Mucina@uwa.edu.au (corresponding author).

*** Department of Geography \& Environmental Studies. Stellenbosch University, Private Bag X1. Matieland 7602, Stellenbosch, South Africa.
} 
by regional/local endemics (e.g. Centaurea sterilis, Cephalaria coriacea, Elytrigia reflexiaristatus subsp. strigosus, Euphorbia petrophila, Seseli lehmannii) showing evolutionary relationships with either Pontic or Mediterranean flora. With the increase of altitude, number of endemic species increases while the number of Pontic elements decreases. At these altitudes one can find even some (sub)boreal elements (Antennaria dioica) and species typical of high-altitudes (Hypericum linarioides, Linum jailicola, Ranunculus oreophilus, Veronica gentianoides, Viola oreades), as well as Crimean endemics such as Androsace villosa subsp. taurica, Draba cuspidata, Pulsatilla halleri subsp. taurica, Tephroseris integrifolia subsp. jailicola.

According to the data published by DIDUKH (1983) the Crimean dry grasslands contain, across the whole spectrum of so far described vegetation units, Bromopsis cappadocica in role of a subdominant and locally also dominant. It is therefore that SAITOV \& MiRKIN (1991) suggested classifying the Crimean steppes as a unique order - the 'Bromopsietalia cappadocicae'. The ecological and biogeographical uniqueness of this syntaxonomic solution is pending further enquiry, however at this stage it does not appear that this step has been justified considering Europe-wide variability of the Festuco-Brometea. The invalidly published concept of the Bromopsietalia cappadocicae Saitov et Mirkin 1996 (ICPN Arts. 2b \& 5 ) should be rather considered as synonyms partly of the Stipo pulcherrimae-Festucetalia pallentis Pop 1968 and partly of the Festucetalia valesiacae Soó 1947 (Mucina \& al., unpubl. data). It also appears that the taxonomic concept of 'Bromopsis cappadocica' in Crimea has been misapplied and that in fact the taxon is actually Bromopsis taurica Slyusarenko, currently recognised as valid by the Euro+Med PlantBase (www.emplantbase.org).

The steppes of the Crimea are differentiated into three alliances (DidukH, 1983): (1) The ' $\mathrm{Ca}$ rici humilis-Androsacion' comprises the primary rocky meadow steppes of the yailas (mountain pastures) in the western and central parts of the Crimean Mts at altitudes of 300-1500 m asl (see Figure 1); (2) The yailas of the eastern Crimean
Mts (at altitudes of spanning 600-800 m) support communities of the Adonidio-Stipion tirsae occur (Didukh, 1983; Didukh \& VAKARENKo, 1984; VAKARENKo 1987). (3) The steppes of the Veronicio multifidae-Stipion ponticae (Fig. 1) occur on rich chernozems and stony eroded soils characteristic of the piedmonts of the Crimean Mts.

All three alliances have been well characterised (DiDuKh, 1983). However, because of nomenclature inconsistencies, they remain invalidly described. This paper is aimed at rectifying this situation and present validations of these alliances as well as they type associations.

As an addendum, we also validate some other (herb-rich and scrub) syntaxa from Ukraine.

\section{VALIDATION OF THE CRIMEAN STEPPE ALLIANCES}

The validation procedures follow the principles of the International Code of Phytosociological Nomenclature (ICPN; WeBER \& al., 2000). The nomenclature of plant taxa follows Euro +Med PlantBase (www.emplantbase.org) in most cases. In order to facilitate the nomenclatural understanding, we also included an Appendix 1 fea turing a crosswalk between traditional Ukrainian taxonomic usage and the current Euro+Med nomenclature and taxonomy.

We present here type relevés of those associations that serve as the type associations of the validated alliances. In the relevés the numerical values are in \%, except for the category 1 , which means that the projected cover was less than $1 \%$, and category $r$ which indicates that one specimen of the species was recorded in the plot.

\section{Androsaco tauricae-Caricion humilis Didukh} all. nov. hoc loco

(Stipo pulcherrimae-Festucetalia pallentis, Festuco-Brometea)

Synonym: Carici humilis-Androsacion Didukh 1983 (ICPN Arts. 2b \& 5)

Holotypus hoc loco: Genisto albidae-Stipetum lithophilae Didukh et Mucina 2014 (see below).

Diagnostic taxa: Androsace villosa subsp. taurica, Asperula supina, Carex humilis, Cytisus hir- 
sutus subsp. polytrichus, Draba cuspidata, Festuca callieri, Genista albida, Hypericum linarioides, Potentilla taurica, Sideritis taurica, Teucrium montanum subsp. montanum, Thymus cherlerioides, Veronica orientalis.
These are typical Crimean subxeric steppes, dominated by graminoids and low shrubs. They are found at low altitudes and seldom also at midaltitudes (900-1400 m asl) on plateau-like yailas. Carex humilis, Festuca callieri, Stipa pennata

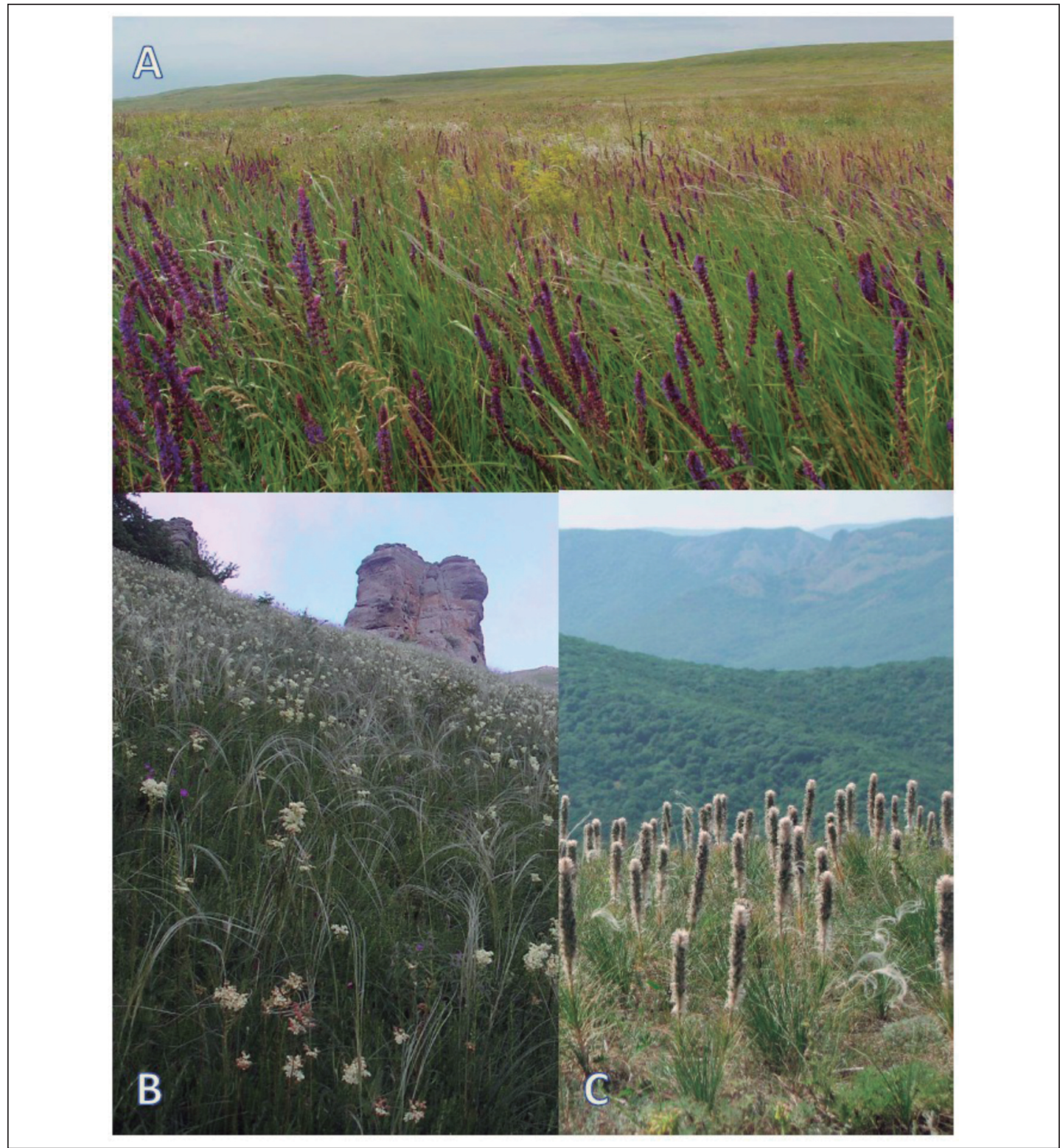

Figure 1. - Selected images of the Crimea steppes: A: Salvia nemorosa, Galium verum and Stipa zalesskii subsp. pontica dominated steppe of the Veronici multifidae-Stipion ponticae on the Kerch Peninsula. B: Rocky steppe stands of the Androsaco tauricae-Caricion humilis on South Demerdzhi (alt. $950 \mathrm{~m}$ ) near Alushta. The aspect is dominated by Stipa pennata subsp. lithophila and Filipendula vulgaris. C: A stand of the Bromopsido tauricae-Asphodelinetum tauricae (Veronicio multifidae-Stipion ponticae) near Sudak, with prominent formed by Asphodeline taurica. All photos: Y.P. Didukh. 
subsp. lithophila are the dominating graminoids while Thymus cherlerioides, T. roegneri and $\mathrm{He}$ lianthemum canum subsp. stevenii are frequent sub-shrubs (DiDUKH, 1993). Soils are rocky rendzina, often much eroded, as well as carbonate chornozem-like soils with high carbonate (7$10 \%$ ) and humus status (6-10\%; DrAGAN, 2004).

\section{Potentillo tauricae-Caricetum humilis ass. nov. hoc loco}

Synonym: Potentilletum depressae Didukh 1983 (ICPN Arts. 2b \& 5).

Holotypus hoc loco: Ukraine: Crimean, Yalta City Council, Yalta Mountain-Forest Natural Reserve, Yaila Ai-Petri. Lat. 44.4813 N, 34.0397 E; Altitude: $1215 \mathrm{~m}$ asl. Substrate: skeletal, mountain black soil with outcrops of limestone (20\%); Vegetation cover: $75 \%$; Vegetation height: $60 \mathrm{~cm}$ (max), $20 \mathrm{~cm}$ (average); Sampled by Y.P. Didukh (75018) on 9 June 1975.

Carex humilis 30, Festuca callieri 20, Filipendula vulgaris 10, Medicago falcata 10 , Thymus cherlerioides 10, Teucrium chamaedrys 5, Trifolium ambiguum 5, Bromopsis taurica 3, Alopecurus vaginatus 3, Thymus roegneri 3, Hypericum linarioides 2, Allium rupestre 1, Alyssum repens subsp. trichostachyum 1, Asperula supina 1, Bupleurum falcatum subsp. cernuum 1, Cruciata taurica 1, Euphorbia petrophila 1, Helichrysum graveolens 1 , Hieracium tephrocephalum 1, Klasea radiata 1, Koeleria macrantha 1, Phlomis herba-venti subsp. pungens 1, Pilosella bauhinii 1, Plantago lanceolata 1, Potentilla taurica 1, Teucrium montanum subsp. montanum 1 , Thalictrum minus 1 , Veronica orientalis 1, Ajuga orientalis r, Draba cuspidata r, Carex otrubae r, Minuartia setacea r, Ranunculus oreophilus r, Thesium brachyphyllum $\mathrm{r}$, Trinia glauca $\mathrm{r}$.

Genisto albidae-Stipetum lithophilae ass. nov. hoc loco

Synonym: Stipetum lithophilae Didukh 1983 (ICPN Arts. 2b \& 5)

Holotypus hoc loco: Ukraine: Crimea, Yalta City Council, Yalta Mountain Forest Natural Reserve, Yaila Ai-Petri. Lat. 44.4472 N, 34.0474 E; Altitude: $1130 \mathrm{~m}$ asl; Substrate: heavily eroded soil, with outcrops of carbonates $(30-40 \mathrm{~cm})$; Ve- getation cover: $70 \%$; Vegetation height: $1 \mathrm{~m}$ (max), $60 \mathrm{~cm}$ (average); Sampled by Y.P. Didukh (rel. 80236) on 22 June 1980.

Stipa pennata subsp. lithophila 30, Genista albida 30, Carex humilis 20, Thymus roegneri 30, Helianthemum canum subsp. stevenii 5, Thymus cherlerioides 5, Alopecurus vaginatus 3, Bromopsis taurica 3, Paronychia cephalotes 3, Scorzonera crispa 3, Sideritis taurica 3, Thalictrum minus 3, Teucrium chamaedrys 3, Asperula supina 2, Elymus reflexiaristatus subsp. strigosus 2, Potentilla taurica 2, Alyssum repens subsp. trichostachyum 1, A. tortuosum 1, Androsace villosa subsp. taurica 1 , Anthyllis vulneraria subsp. pulchella 1, Cephalaria coriacea 1, Convolvulus calverti 1, Cytisus hirsutus subsp. polytrichus 1, Erysimum cuspidatum 1, Euphorbia petrophila 1, Festuca callieri 1, Filipendula vulgaris 1, Galium album 1, Koeleria macrantha 1, Linum tenuifolium 1,Pedicularis sibthorpii 1, Pulsatilla halleri subsp. taurica 1, Teucrium montanum subsp. montanum 1, Viola ambigua 1, Campanula sibirica r, Draba cuspidata r, Iberis saxatilis r, Seseli lehmannii $\mathrm{r}$.

\section{Adonido vernalis-Stipion tirsae Didukh all. nov. hoc loco}

(Festucetalia valesiacae, Festuco-Brometea)

Synonym: Adonido vernalis-Stipion tirsae Didukh 1983 (ICPN Arts. 2b \& 5).

Holotypus hoc loco: Adonido-Stipetum tirsae Didukh in Didukh et Mucina 2014 (see below)

Diagnostic taxa: Adonis vernalis, Cerastium biebersteinii, Elytrigia intermedia subsp. trichophora, Paeonia tenuifolia, Phlomis herba-venti subsp. pungens, Poa angustifolia, Stipa tirsa, Trinia glauca.

The Adonido vernalis-Stipion tirsae includes the Crimean meadow-steppe communities of the plateau-like eastern yailas at the altitudes spanning 700-1000 $\mathrm{m}$. These are communities dominated by tussock-forming grasses such as Festuca valesiaca and Stipa tirsa, short-rhizome grasses (Bromopsis taurica and Poa angustifolia), and low-grown shrubs such as Genista albida, Helianthemum stevenii and Thymus roegneri. 
The relief of the yailas is characterised by high level of karst formation with deep mesic soils in depressions. The mean annual temperature at these altitudes spans $6.3-6.5^{\circ} \mathrm{C}$ (the average temp. in January and July is $3.4^{\circ} \mathrm{C}$ and $16^{\circ} \mathrm{C}$, resp.) and the mean annual precipitation attains $550-600 \mathrm{~mm}$ of which most falls during the period March-October (VED, 2000). Because of high level of karst relief surface water drains quickly. These conditions lead to formation of chernozem-like soils and calcareous chernozem-like rendzinas, characterised by the high levels of soil humus (6-20\%; DRAGAN, 2004).

\section{Adonido vernalis-Stipetum tirsae ass. nov. hoc loco}

Synonym: Adonido-Stipetum tirsae Didukh 1983 (ICPN Arts. 2b \& 5)

Holotypus hoc loco: Ukraine: Crimea, Belogorsky District, Karabi Yaila. 44.8898249 N 34.570713 E; Altitude: $850 \mathrm{~m}$ asl; Aspect: E; Slope: $10^{\circ}$; Substrate: mountain black soil; Vegetation cover: $100 \%$; Vegetation height: $1 \mathrm{~m}$ (max), $50 \mathrm{~cm}$ (average); Sampled by Y.P. Didukh (rel. 80244) on 25 June 1980.

Stipa tirsa 50, Festuca stricta subsp. sulcata 40, Thymus kosteleckyanus 10, Galium album 5, Filipendula vulgaris 5, Koeleria macrantha 5, Helianthemum canum subsp. stevenii 3, Poa angustifolia 3, Achillea setacea 1, Adonis vernalis 1, Anthyllis vulneraria subsp. pulchella 1, Cerastium biebersteinii 1, Carex caryophyllea 1, Securigera varia 1, Euphorbia agraria 1, E. nicaeensis subsp. stepposa 1, Fragaria viridis 1, Geranium sanguineum 1 , Helianthemum nummularium subsp. ovatum 1, Hieracium sp. 1, Hypericumlinarioides 1, Inula ensifolia 1, Inula oculus-christi 1, Paeonia tenuifolia 1, Phlomis herba-venti subsp. pungens 1, Plantago lanceolata 1 , Potentilla recta 1 , Sanguisorba minor subsp. balearica 1 , Prunella laciniata 1, Pulsatilla halleri subsp. taurica 1, Ranunculus illyricus 1, Teucrium chamaedrys 1 , Teucrium capitatum 1, Trifolium medium 1, T. montanum 1, Veronica incana 1 , Allium rotundum $\mathrm{r}$, Arenaria serpyllifolia r, Clinopodium acinos r, Helictochloa compressa $\mathrm{r}$, Lathyrus pannonicus $\mathrm{r}$.

Veronico multifidae-Stipion ponticae Didukh all. nov. hoc loco

(Festucetalia valesiacae, Festuco-Brometea)
Synonym: Veronico multifidae-Stipion ponticae Didukh 1983 (ICPN Arts. 2b \& 5)

Holotypus hoc loco: Bromopsido tauricae-Stipetum ponticae Didukh in Didukh et Mucina 2014 (see below).

Diagnostic taxa: Aegilops triuncialis, Agropyron cristatum subsp. ponticum, Asperula tenella, Asphodeline taurica, Centaurea orientalis, Convolvulus cantabrica, Crupina vulgaris, Galium biebersteinii, Onobrychis arenaria subsp. miniata, Onosma rigida, Polygala major, Stipa zalesskii subsp. pontica, Veronica multifida subsp. capsellicarpa.

These are the typical Crimean xeric steppes, dominated by grasses) and low shrubs. They are found in the low-altitude (seldom also in the middle-altitude) mountain belts, at altitudes up to $450 \mathrm{~m}$ (seldom up to $600 \mathrm{~m}$ ) on the north-facing macroslope. Occasionally (in fragments) they are also found on the south-facing macroslope. Most these steppes are found on plateaus (Bromopsido tauricae-Stipetum ponticae) or on moderate slopes (not more than $30^{\circ}$ ) with shallow, erosion-disturbed soils (Bromopsido tauricae-Asphodelinetum tauricae).

The climate of the region of the Veronico multifidae-Stipion ponticae is having submediterranean features (warm summers and mild winters) as well as continental temperate climate. The mean annual temperature is between $10^{\circ} \mathrm{C}$ and $11.7^{\circ} \mathrm{C}$ and the mean temperatures in July spans $22-24^{\circ} \mathrm{C}$. The annual precipitation spans $300-550$ $\mathrm{mm}$, most of which falls in autumn and spring. Summers are markedly dry (VED, 2000). Chernozem soils supporting these steppes on the northern macroslope have a high carbonate (7-10\%) and humus status $(3-15 \%)$. The brown soils on the south-facing have lower humus content (1.5$6 \%$; DraGAN, 2004). Floristically, the communities of this alliance are similar to the Stipa lessingiana communities that are common to the northern plains of the Crimea (DIDUKH, 1992).

\section{Bromopsido tauricae-Stipetum ponticae ass. nov. hoc loco}

Synonym: Elytrigio-Stipetum ponticae Didukh 1983 (ICPN Arts. 2b \& 5).

Holotypus hoc loco: Ukraine: Crimea, Symferopilsky District, Donskoe. 45.0375655 N, 34.2062759 
E; Altitude: $300 \mathrm{~m}$ asl; Relief: flap top of low ridge; Substrate: black soil, $20 \mathrm{~cm}$ deep with outcrops of limestone rocks; Vegetation cover: $80 \%$; Vegetation height: $1 \mathrm{~m}$ (max), $60 \mathrm{~cm}$ (average); Sampled by Y.P. Didukh (rel. 80076) on 3 June 1980.

Stipa zalesskii subsp. pontica 60, Poa sterilis 30 Festuca valesiaca 10, Bromopsis taurica 3, Filipendula vulgaris 3, Salvia nutans 3, Thymus roegneri 1, Adonis vernalis 1, Prunus tenella 1, Asperula tenella 1, Bellevalia speciosa 1, Centaurea scabiosa subsp. adpressa 1, Cleistogenes serotina 1, Convolvulus cantabrica 1, Eryngium campestre 1, Euphorbia nicaeensis subsp. stepposa 1, Falcaria vulgaris 1, Ferulago campestris 1, Galium album 1, G. biebersteinii 1, Helichrysum arenarium 1, Koeleria macrantha 1, Leontodon biscutellifolius 1, Onobrychis arenaria 1, Onosma cinerea 1, Pimpinella peregrina 1, Polygala major 1, Potentilla recta 1, Sanguisorba minor subsp. balearica 1, Stachys recta 1, Teucrium capitatum 1, T. chamaedrys 1, Thalictrum minus 1, Vinca herbacea 1, Viola ambigua $1, \mathrm{Ce}$ rinthe minor r, Gypsophila glomerata r, Jurinea roegneri r, Medicago minima $\mathrm{r}$, Scabiosa taurica $\mathrm{r}$, Trinia glauca $\mathrm{r}$.

\section{Bromopsido tauricae-Asphodelinetum tauricae ass. nov. hoc loco}

Synonym: Asphodelinetum tauricae Didukh 1983 (ICPN Arts. 2b \& 5).

Holotypus hoc loco: Ukraine: Crimea, Kirov District, Stary Krym, Agarmysh Mt. 45.03416 N, 35.05076 E; Altitude: $540 \mathrm{~m}$ asl; Aspect: 180 ; Slope: $25^{\circ}$; Substrate: black soil, eroded in places and exposing limestone outcrops; Vegetation cover: $60 \%$; Vegetation height: $1 \mathrm{~m}(\max ), 30 \mathrm{~cm}$ (average). Sampled by Y.P. Didukh (rel. 78707) on 16 June 1978.

Asphodeline taurica 25, Artemisia alpina 20, Bromopsis taurica 3, Festuca stricta subsp. sulcata 3, Helianthemum canum subsp. stevenii 3, Thymus roegneri 3, Alyssum repens subsp. trichostachyum 2, Pimpinella tragium subsp. lithophila 2, Agropyron cristatum subsp. ponticum 1, Anthyllis vulneraria subsp. boissieri 1, Asperula tenella 1, Bunium ferulaceum 1, Carex caryophyllea 1, Centaurea orientalis 1, Cephalaria coriacea 1, Cleistogenes serotina 1, Cota tinctoria 1, Di- chanthium ischaemum 1, Erysimum cuspidatum 1, Eryngium campestre 1, Euphorbia petrophila 1, Fumana procumbens 1, Galium glaucum 1, Geranium sanguineum 1 , Jurinea roegneri 1 , Koeleria macrantha 1, Leontodon biscutellifolius 1 , Linum hirsutum L. subsp. hirsutum 1, L. tenuifolium 1, Medicago falcata 1, Muscari neglectum 1, Paronychia cephalotes 1, Phlomis herba-venti subsp. pungens 1, Poa bulbosa 1, Prunus tenella 1, Salvia scabiosifolia 1, Sideritis taurica 1, Silene densiflora 1, Stipa pulcherrima 1, Teucrium chamaedrys 1, Veronica multifida subsp. capsellicarpa 1, Allium sp. r, Alyssum alyssoides r, Arenaria serpyllifolia $\mathrm{r}$, Crupina vulgaris $\mathrm{r}$, Ferulago campestris $\mathrm{r}$, Iris pumila $\mathrm{r}$, Potentilla recta $\mathrm{r}$, Scorzonera crispa r, Sedum acre r, S. hispanicum $\mathrm{r}$, Thesium brachyphyllum $\mathrm{r}$, Vinca herbacea $\mathrm{r}$.

\section{Melico tauricae-Helianthemetum stevenii ass. nov. hoc loco}

Synonym: Melico tauricae-Paeonietum tenuifoliae Didukh 1983 (ICPN Arts. 2b \& 5).

Holotypus hoc loco: Ukraine: Crimea, Sevastopol City Council, Shyroke-Peredove, Samnalyk Mt. Lat. 44.4838 N, Long. 33.7740 E; Altitude: $450 \mathrm{~m}$ asl; Habitat: open grassland glade among woodland of Juniperus excelsa; Substrate: brown soil, locally eroded and exposing outcrops of limestone; Aspect: $180^{\circ}$; Slope: $15^{\circ}$; Vegetation cover: 50\%; Lichen cover: 20\%; Cover of surface rocks: 25\%; Vegetation height: $60 \mathrm{~cm}(\max ), 30$ cm (average); Sampled by Y.P. Didukh (rel. 80197) on 19 June 1980.

Thymus roegneri 30, Helianthemum canum subsp. stevenii 20, Poa sterilis 5, Teucrium chamaedrys 5, Bromopsis taurica 3, Fumana procumbens 3, Melica taurica 2, Teucrium capitatum 2, Potentilla pedata 1, Anthyllis vulneraria subsp. boissieri 1, Carex caryophyllea 1, Convolvulus cantabrica 1, Elytrigia caespitosa subsp. nodosa 1, Eryngium campestre 1, Euphorbia petrophila 1, Festuca stricta subsp. sulcata 1, Galium album 1, Inula oculus-christi 1, Iris pumila 1, Koeleria lobata 1, Muscari racemosum 1, Paeonia tenuifolia 1, Phlomis herba-venti subsp. pungens 1, Pimpinella tragium subsp. lithophila 1, Tanacetum corymbosum 1, Scorzonera crispa 1, Veronica multifida subsp. capsellicarpa 1, Asperula creta- 
cea $\mathrm{r}$, Campanula sibirica $\mathrm{r}$, Centaurea sterilis $\mathrm{r}$, Euphorbia myrsinites $\mathrm{r}$, Linum austriacum $\mathrm{r}, \mathrm{Me}$ dicago minima $\mathrm{r}$, Ornithogalum pyrenaicum $\mathrm{r}$. Lichens: Cladonia rangiferina 10, Cladonia sp. 3.

\section{VALIDATION OF OTHER SUBMEDITE- RRANEAN CRIMEAN SYNTAXA}

\section{'Diantho humilis-Velesion rigidae'}

The 'Diantho humilis-Velesion' of KorZHENEVSKII (1990) and KoRZHENEVSKII \& KLYUKIN (1990) was not validly described because there was no validly described association assigned to the alliance in the protologue. The authors selected nomenclature types for all four subassociations of the 'Diantho humilis-Velesietum' (see fine printed text below the table on page 96), however, they failed to assign unequivocally the nomenclatural type of the association. Their suggestion that the "subassociation D.-V. elytrigietosum should be considered the type of the association" (note: 'D.-V.' means 'Diantho humilis-Velesietum') is not admissible from the nomenclatural point of view. It is therefore I assign here a relevé to become the holotypus and hence validate the name Diantho humilis-Velezietum rigidae whole the latter then become the holotypus of the Diantho humilis-Velezion rigidae:

\section{Diantho humilis-Velezietum rigidae Korzhe-} nevskii et Klyukin ex Didukh et Mucina ass. nova hoc loco

Synonyms: 'Diantho humilis-Velesietum rigidae' Korzhenevskii 1990 (ICPN Art. 2b); 'Diantho humilis-Velesietum rigidae' Korzhenevskii et Klyukin 1990 (ICPN 2b \& 5).

Holotypus hoc loco: KORZHENEVSKII \& KLYUKIN (1990: rel. 15, Table pags. 92-96).

\section{Diantho humilis-Velezion rigidae Korzhenevskii et Klyukin ex Didukh et Mucina all. nov. hoc loco (order uncertain, Stipo-Trachynietea distachyae) \\ Synonyms: 'Diantho humilis-Velesion rigidae' Korzhenevskii 1990 (ICPN Art. 8); 'Diantho hu- milis-Velesion rigidae' Korzhenevskii et Klyukin 1990 (ICPN Art. 5).}

Holotypus hoc loco: Diantho humilis-Velezietum rigidae Korzhenevskii et Klyukin ex Didukh et Mucina 2014 (this paper)

\section{'Elytrigio nodosae-Rhoion coriariae'}

The 'Elytrigio nodosae-Rhoion coriariae' (recte: Elytrigio nodosae-Rhuion coriariae') was described by KORZHENEVSKII \& RIFF (2002) who assigned two associations to this alliance: the $R a$ pistro rugosi-Melicetum tauricae Korzhenevskii et Riff 2002 and the Meliloto taurici-Seselietum dichotomi Korzhenevskii et Riff 2002. The names of these both association as well as of the alliance were, however, invalidly described because the authors failed to identify the holotypes of these syntaxa in expressis verbis - a requirement of the Article 5 of the IPCN ( $3^{\text {rd }}$ ed.).

The names of the associations are also illegitimate according to ICPN Art. 29b since the despite both associations (see above) are actually scrub vegetation (hence two-layered at least) and none of the eponymous species is a shrub.

Here we validate these syntaxa as follows:

Melico tauricae-Rhuetum coriariae ass. nov. hoc loco

Holotypus hoc loco: KoRZHENEVSKII \& RIFF (2002: Table 3, rel. 1).

Synonym: Rapistro rugosi-Melicetum tauricae Korzhenevskii et Riff 2002 (ICPN Art. 3k, 5 \& 29b)

Seseli dichotomi-Rhuetum coriariae ass. nov. hoc loco

Holotypus hoc loco: KoRzHENEVSKII \& RIFF (2002: Table 4, rel. 3)

Synonym: Rapistro rugosi-Melicetum tauricae Korzhenevsky et Ryff 2002 (ICPN Arts. 3k, 5 \& 29b).

Elytrigio nodosae-Rhuion coriariae Korzhenevskii et Riff ex Didukh et Mucina all. nov. hoc loco

(Paliuretalia, Rhamno-Prunetea)

Synonym: 'Elytrigio nodosae-Rhoion coriariae' Korzhenevskii et Riff 2002 (ICPN Art. 5)

Holotypus hoc loco: Melico tauricae-Rhuetum coriariae Didukh et Mucina 2014 (this paper, see above) 
Diagnostic species: Elymus nodosus, Melica taurica, Melilotus tauricus, Rhus coriaria .

This alliance accommodates low submediterranean scrub on marl slopes of Crimea and should be classified not within the Onosmo polyphyllae-Ptilostemonetea Korzhenevskii 1990 (recte: Drypidetea spinosae Quézel 1964), but rather within the
Paliuretalia Trinajstić 1978 (Rhamno-Prunetea Rivas Goday et Borja Carbonell ex Tx. 1962).

\section{ACKNOWLEDGEMENTS}

We thank Thomas Raus (Berlin) for clarifying the nomenclature and taxonomy of some plant taxa. Jean-Paul Theurillat kindly commented on several nomenclature issues.

\section{REFERENCES}

Didukh, Y.P. - 1983 - Opyt klassifikatsii kserofilnoi polukustarnichkovoi i travyanistoi rastitel'nosti Gornogo Kryma (An attempt of classification of xerophilous semi-scrub and grassland vegetation of the Gorny Krym) - Bot. Zhurn. 68: 1456-1466. (In Russian).

Didukh, Y.P. - 1992 - Rastitelny pokrov Gornogo Kryma (struktura, dinamika, evolyutsyya, okhrana) (Vegetation cover of the Gorny Krym (structure, dynamics, evolution, protection) - Naukova Dumka, Kyiv. (in Ukrainian).

Didukh, Y.P. \& Shelyag-Sosonko, Y.R. - 2003 - Geobotanichne rayonuvannya Ukrainy ta sumizhnykh terytoriy (geobotanical division of Ukraine and neighboring territories) - Ukr. Bot. Zhurn. 60: 6-17. (in Ukrainian)

Didukh, Y.P. \& Vakarenko, L.P. - 1984 - Porivnyalnyi analiz syntaxoniv florystychnoi klasyfikatsiyi stepiv i tomilyariv Girskogo Krymu (Comparative analysis of the syntaxa of floristic classification of steppes and tomillares of the Gorny Krym) - Ukr. Bot. Zhurn. 41: 11-19. (in Ukrainian).

Dragan, N.A. - 2004- Pochvennyye resursy Kryma (Soil resources of Crimea) - Dolya, Simpferopol. (in Russian)

Isachenko, T.I. \& Lavrenko, E.M. - 1980 - Rastitel'nost' Evropeiskoi chasti SSSR. Botaniko-geograficheskoye raionirovanie (Vegetation of the European part of the Soviet Union. Botanico-geographical regional classification) Leningrad, Nauka. (In Russian).

Korzhenevskii, V.V. - 1990 - Sintaksonomicheskii sostav rastitel'nosti flyshebogo nizkogor'ya yugo-istochnogo
Kryma (Syntaxonomic structure of the vegetation of flysch ranges of the south-eastern Crimea) - Sborn. Nauch. Trud. Yalta 110: 80-90. (In Russian).

Korzhenevskii, V.V. \& Klyukin, A.A. -1990- O sintaksonakh-indikatorakh sklonovykh protsesov (On syntaxonomic indicators of slope processes) - Sborn. Nauch. Trud. Yalta 110: 90-103. (In Russian).

Korzhenevskii, V.V. \& Riff, L.E. - 2002- O novykh sintaksonakh i ob'eme klassa Onosmo polyphyllae-Ptilostemetea (On new syntaxa and the concept of the Onosmo polyphyllae-Ptilostemetea class) - Visti Biosfernogo Zapovidnika 'Askaniya-Nova' 4: 20-29. (In Russian).

Saitov, M.S. \& Mirkin, B.M. - 1991 - O vysshikh edinitsakh sintaksonomii stepei klassa Festuco-Brometea Br.B1. et Tx. 43 na territorii SSSR (On high-rank syntaxa of the steppe vegetation of the Festuco-Brometea Br.-B1. et Tx. 43 on the territory of Soviet Union) - Biul. Mosk. Obshch. Ispyt. Prir., Odt. Biol. 96: 87-98.

Vakarenko, L.P., Shelyag-Sosonko, Y.R. \& Didukh Y.P. 1987 - Rastitel'nost' predgornogo Kryma i ego botaniko-geograficheskoe raionirovanie (The foothill vegetation of Crimea and its botanico-geographical zonation) - Bot. Zhurn. 72: 39-48. (in Russian).

Ved, I.P. - 2000 - Klimaticheskii atlas Kryma (Climate atlas of Crimea) - Tavriya-Plyus, Simferopol. (In Russian).

Weber, H.E., Moravec, J. \& Theurillat, J.-P. -2000 - International Code of Phytosociological Nomenclature. 3rd edition - J. Veg. Sci. 11: 739-768. 


\section{APPENDIX 1}

Nomenclatural (and taxonomic) crosswalk between the traditional Ukrainian usage and the nomenclature as currently fetaured in Euro+med PlantBase.

\begin{tabular}{|c|c|}
\hline Ukrainian nomenclatural tradition & Euro+Med PlantBase (www.emplantbase.org) \\
\hline Acinos arvensis (Lam.) Dandy & Clinopodium acinos (L.) Kuntze \\
\hline Agropyron ponticum (Nevski ) Tzvelev & Agropyron cristatum (L.) Gaertner \\
\hline Allium jailae Vved. & Allium scorodoprasum subsp. jailae (Vved.) Stearn. \\
\hline Alyssum trichostachyum Rupr. & Alyssum repens subsp. trichostachyum (Rupr.) Hayek \\
\hline Amygdalus nana $\mathrm{L}$. & Prunus tenella Batsch \\
\hline Androsace taurica Ovcz. & Androsace villosa subsp. taurica (Ovcz.) Fed. \\
\hline Anthemis tinctoria $\mathrm{L}$. & Cota tinctoria (L.) J. Gay \\
\hline Anthyllis biebersteinii Popl. & Anthyllis vulneraria subsp. pulchella (Vis.) Bornm. \\
\hline Anthyllis taurica Juz. & Anthyllis vulneraria subsp. boissieri (Sagorski) Bornm. \\
\hline Artemisia caucasica Willd. & Artemisia alpina Willd. \\
\hline Artemisia repens Pall. ex Willd. & Artemisia austriaca Jacq. \\
\hline Asperula caespitans Juz. & Asperula supina subsp. caespitans (Juz.) S. Pyatunina \\
\hline Asperula galioides M. Bieb. & Galium glaucum $\mathrm{L}$. \\
\hline Asperula vestita V. I. Krecz. & Asperula cretacea Willd. \\
\hline Asperula stevenii V. I. Krecz. & Asperula tenella Degen \\
\hline Bellevalia sarmatica (Pall. ex Georgi) Woronow & Bellevalia speciosa Woronow ex Grossh. \\
\hline Botriochloa ischaemum (L.) Keng & Dichanthium ischaemum (L.) Roberty \\
\hline Bupleurum exaltatum M. Bieb. & Bupleurum falcatum subsp. cernuиm (Ten.) Arcang. \\
\hline Campanula taurica Juz. & Campanula sibirica $\mathrm{L}$. \\
\hline Centaurea adpressa Ledeb. & Centaurea scabiosa subsp. adpressa (Ledeb.) Gugler \\
\hline Cephalaria coriacea (Willd.) Steud. & Cephalaria coriacea (Willd.) Roem. \& Schult. ex Steud. \\
\hline Chamaecytisus polytrichus (M. Bieb.) Rothm. & Cytisus hirsutus subsp. polytrichus (M. Bieb.) Hayek \\
\hline Convolvulus bracteosus Juz. & Convolvulus calvertii Boiss. \\
\hline Coronilla varia $\mathrm{L}$. & Securigera varia $(\mathrm{L}$.$) Lassen$ \\
\hline Crinitaria villosa (L.) Cass. & Galatella villosa (L.) Rchb. f. \\
\hline Elytrigia nodosa (Nevski) Nevski & Elymus nodosus (Nevski) Melderis \\
\hline Elytrigia strigosa (M. Bieb.) Nevski & Elymus reflexiaristatus subsp. strigosus (M. Bieb.) Melderis \\
\hline Elytrigia triochophora (Link) Nevski & Elymus hispidus subsp. barbulatus (Schur) Melderis \\
\hline Euphorbia stepposa Zoz ex Prokh. & Euphorbia nicaeensis subsp. stepposa (Zoz) Greuter \& Burdet \\
\hline Ferulago taurica Schischk. & Ferulago campestris (Besser) Grec. \\
\hline Festuca rupicola Heuff. & Festuca stricta subsp. sulcata (Hack.) Patzke ex Joch. Müll. \\
\hline Helianthemum stevenii Rupr. ex Juz. \& Pozdeeva & $\begin{array}{l}\text { Helianthemum canum subsp. stevenii (Rupr. ex Juz. \& Pozd.) } \\
\text { M. Proctor }\end{array}$ \\
\hline Helictotrichon tauricum Prokud. & Helictochloa compressa (Heuff.) Romero Zarco \\
\hline Hieracium bauhinii Besser & $\begin{array}{l}\text { Pilosella piloselloides subsp. bauhinii (Schult.) S. Bräut. \& } \\
\text { Greuter }\end{array}$ \\
\hline Hieracium tephropodum (Zahn) Üksip & Hieracium tephrocephalum Vuk. ex Nyman \\
\hline Hypericum tauricum $\mathrm{R}$. Keller & Hypericum linarioides Bosse \\
\hline Inula aspera Poir. & Inula salicina subsp. aspera (Poir.) Hayek \\
\hline Jurinea sordida Steven & Jurinea roegneri $\mathrm{K}$. Koch. \\
\hline Kengia serotina (L.) Packer & Cleistogenes serotina $(\mathrm{L}$.$) Keng.$ \\
\hline Koeleria cristata (L.) Pers. & Koeleria macrantha (Ledeb.) Schult. \\
\hline Leontodon asper (Waldst. \& Kit.) Poir. & Leontodon biscutellifolius DC. \\
\hline
\end{tabular}


Ukrainian nomenclatural tradition

Linum lanuginosum Juz.

Onosma taurica Pall.

Ornithogalum flavescens Lam.

Otites densiflora (d'Urv.) Grossh.

Phlomis taurica Hartwiss ex Bunge

Pimpinella lithophila Schischk.

Pimpinella taurica (Ledeb.) Steud.

Potentilla depressa Will. ex Schlecht.

Poterium polygamum Waldst. \& Kit.

Pulsatilla taurica Juz.

Pyrethrum corymbosum (L.) Scop.

Rumia crithmifolia (Willd.) Koso-Pol.

Senecio jailicola Juz.

Serratula radiata (Waldst. \& Kit.) M. Bieb.

Sideritis comosa (Rochel ex Benth.) Stank.

Sideritis taurica Steph. ex Willd.

Stipa pontica P. Smirn.

Stipa litophila P. Smirn.

Teucrium jailae Juz.

Teucrium polium sensu auct. ucrain., non L.

Thymus callieri Bord. ex Velen.

Thymus dzevanovskyi Klokov \& Des.-Shost.

Thymus hirsutus M. Bieb.

Thymus tauricus Klokov \& Des.-Shost.

Veronica capsellicarpa Dubovik

Veronica taurica Willd.
Euro+Med PlantBase (www.emplantbase.org)

Linum hirsutum L. subsp. hirsutum

Onosma cinerea Schreb.

Ornithogalum pyrenaicum L.

Silene densiflora d'Urv.

Phlomis herba-venti subsp. pungens (Willd.) Maire ex

DeFilipps

Pimpinella tragium subsp. lithophila (Schischk.) Tutin

Pimpinella peregrina $\mathrm{L}$.

Potentilla microphylla var. depressa Wall. ex Lehm.

Sanguisorba minor subsp. balearica (Nyman) Muñoz Garm.

\& C. Navarro

Pulsatilla halleri subsp. taurica (Juz.) K. Krause

Tanacetum corymbosum (L.) Sch. Bip.

Trinia crithmifolia (Willd.) H. Wolff

Tephroseris integrifolia subsp. jailicola (Juz.) Greuter

Klasea radiata (Waldst. \& Kit.) Á. Löve \& D. Löve

Sideritis montana var. comosa Rochel ex Benth.

Sideritis syriaca $\mathrm{L}$.

Stipa zalesskii subsp. pontica (P.A. Smirn.) Tzvelev

Stipa pennata subsp. lithophila (P. Smirn.) Martinovský

Teucrium montanum L. subsp. montanum

Teucrium capitatum $\mathrm{L}$.

Thymus roegneri $\mathrm{K}$. Koch

Thymus kosteleckyanus Opiz

Thymus cherlerioides Vis.

Thymus cherlerioides Vis.

Veronica multifida subsp. capsellicarpa (Dubovik) Elen.

Veronica orientalis Mill. 